\title{
Gamma Radiosynthesis of Colloidal Silver Nanoparticles Stabilized in I-Carrageenan Under Atmospheric Gases: A Surface Plasmon Resonance Based Study
}

\author{
D. P. Perkasa*, M. Y. Yunus, Y. Warastuti, B. Abbas \\ Center for Isotopes and Radiation Application, National Nuclear Energy Agency (BATAN), \\ Jl. Lebak Bulus Raya No. 49, Jakarta 12440, Indonesia
}

\section{ARTICLE INFO}

Article history:

Received 25 June 2019

Received in revised form 15 July 2020

Accepted 15 September 2020

Keywords:

Gamma radiosynthesis

Silver nanoparticles

t-Carrageenan

Surface plasmon resonance

\begin{abstract}
A B S T R A C T
1-Carrageenan is a biodegradable and biocompatible biomaterial which potentially stabilizes colloidal silver nanoparticles (AgNPs). The present study explored gamma radiosynthesis of AgNPs at varied concentration of 1 -carrageenan solutions. The reaction system contained $1.0 \mathrm{mM}$ silver precursor from silver nitrate salt. Gamma irradiation was conducted at doses up to $20 \mathrm{kGy}$ under simple condition, i.e., atmospheric gases and without addition of hydroxyl radical scavenger. The behavior of AgNPs in suspension was characterized based on their surface plasmon resonance (SPR) absorption spectra which were measured using UV-vis spectrophotometer. The results show that colloidal AgNPs were successfully radiosynthesized due to dual stabilizing/reducing activity of 1-carrageenan. The degradation product of 1-carrageenan shows antioxidant activities, which increase the reducing condition of the reaction system. TEM micrograph reveals that the nanoparticles are spheroid in shape and monodisperse with an average particle size of below $10 \mathrm{~nm}$. The SPR spectra indicate that the highest AgNPs concentration is found for irradiation at a dose of $10 \mathrm{kGy}$ and 1-carrageenan concentration of $1.0 \%(\mathrm{w} / \mathrm{v})$. However, instability of AgNPs occurred a day after radiosynthesis due to oxidative dissolution and agglomeration. Further works on $\mathrm{pH}$ adjustment and optimization on irradiation dose and $\mathbf{l - c a r r a g e e n a n ~ c o n c e n t r a t i o n ~ a r e ~}$ critical to improve the stability of colloidal AgNPs.
\end{abstract}

(C) 2021 Atom Indonesia. All rights reserved

\section{INTRODUCTION}

Silver exhibits unique size-dependent biological, physical and chemical properties at nanoscale which differ greatly from those of bulk materials $[1,2]$. For therapeutic applications in biomedical field, silver nanoparticles (AgNPs) have showed potential antimicrobial [3-5], antioxidant [6], and anticancer [7]. The AgNPs also exhibit strong absorption band in UV-visible (UV-vis) light region due to the surface plasmon resonance (SPR) of conductive electron [8,9] for application in bioimaging and biosensor. The characteristics and behavior of AgNPs are dependent on their particle size and morphology, which are affected by the

*Corresponding author.

E-mail address: dpribadi@batan.go.id

DOI: https://doi.org/10.17146/aij.2021.963 synthesis process $[1,2]$. Therefore, consideration has been given to synthesis of AgNPs to control their morphology and size.

The synthesis of AgNPs in solution usually contains three main components, i.e., metal precursor, reducing agent, and stabilizing agent. Various routes have been developend for the synthesis of AgNPs, which can be categorized as chemical, physical, and biological methods [10]. However, despite the progress that have been made in synthesis techniques, they still have some drawbacks such as the use of toxic reducing agent, high temperature requirement, long processing time, and high energy used while the morphology and particle size are still difficult to control [1,2].

In this context, gamma radiosynthesis offers unique advantages for the synthesis of AgNPs. Firstly, $\mathrm{Ag}^{+}$ions reduced to uncharged state of $\mathrm{Ag}^{0}$ 
by reducing radicals produced by radiolysis of water. Thus, there is no issue about potential toxicity of the residual reducing agent $[3,11]$ which supports the ensuring of its biocompatibility for application in biomedical field. Also, gamma radiosynthesis operates under simple physicochemical conditions that allow the homogenous reduction and nucleation of AgNPs, have the capability of producing AgNPs with narrow particle size distributions, offer effective control of morphology, and can be used to fabricate core-shell and alloyed metal nanoparticles [1,2].

Previous studies about radiosynthesis AgNPs have been reported using different stabilizer such as chitin derivative [11], chitosan [4], honey [3], polyvinyl alcohol [5], and silk fibroin [12]. The use of carrageenan as a stabilizer and reducing agent have been reported by using green processing [13], but not in gamma radiosynthesis yet. The i-carrageenan is sulphated linear polysaccharides extracted from certain species of red algae class (Rhodophyceae) which shows biodegradability and biocompatibility for potential application in biomedical field. Indeed, the l-carrageenan have high metal binding activity [14] which potentially provides steric passivation of radiosynthesized AgNPs. Gamma radiosynthesis of AgNPs is usually conducted in the absence of oxygen and in the presence of $\mathrm{OH}$ radical scavenger (predominantly isopropanol) to prevent unwanted oxidation during the radiosynthesis process $[1,11]$.

The present research studied the synthesis of AgNPs stabilized in $\mathbf{l}$-carrageenan solution using gamma ray radiation. The gamma irradiation was conducted without isopropanol addition and under air atmosphere. The isopropanol was not included in reaction system due to its toxicity to central nerves system [15-17]. Meanwhile, the $\mathrm{N}_{2}$ purging step to remove dissolved oxygen was eliminated because it contributes to longer processing time and additional cost. Beside of the simplicity, the $\mathrm{N}_{2}$ purging facilities is not always available in lab of developing countries.

\section{EXPERIMENTAL METHODS}

\section{Materials}

The $\mathrm{AgNO}_{3}$ (Merck, Darmstad, Germany) and $\mathrm{KBr}$ (Merck, Darmstad, Germany) were of analytical grade and used without further purification. The 1-carrageenan was kindly provided by Research and Development Centre for Marine and Fisheries Product Processing and Biotechnology, Ministry of Marine and Fisheries Affair, Indonesia. Water used in this study was deionized water.

\section{Gamma radiosynthesis of AgNPs}

A fresh $0.2 \mathrm{M} \mathrm{AgNO}_{3}$ solution was prepared by dissolving the silver salt crystal in water at room temperature under dark condition. The 1 -carrageenan solutions were prepared by dissolving l-carrageenan powder in water at $80{ }^{\circ} \mathrm{C}$ for $15 \mathrm{~min}$ under contionous stirring. The solutions were prepared at four level concentration i.e. 0.05, 0.1, 0.5, and $1.0 \%(\mathrm{w} / \mathrm{v})$. The reaction system for gamma radiosynthesis of AgNPs was $200 \mu \mathrm{l}$ of the $0.2 \mathrm{M}$ $\mathrm{AgNO}_{3}$ solution blended with $40 \mathrm{ml}$ of $\mathrm{t}$-carrageenan solution in a screw-capped bottle. The reaction system was homogenized by vortexing for $20 \mathrm{~s}$. There was no nitrogen bubbling treatment nor isopropanol addition subjected to the reaction system.

The reaction system was irradiated using a Co-60 gamma ray irradiator irradiator (series Gammacell 220, MDS Nordion, Ottawa, Canada) at a dose rate of about $5 \mathrm{kGy} / \mathrm{h}$ Center for Application of Isotopes and Radiation-BATAN, Jakarta. The absorbed dose of gamma ray was varied at 5, 10, 15 and $20 \mathrm{kGy}$. The $\mathrm{t}$-carrageenan solution without the addition of $\mathrm{AgNO}_{3}$ was also prepared in the same manner and used as a control.

\section{Characterization of colloidal AgNPs}

Previous studies $[8,9,18]$ reported that UV-vis spectroscopy is one of the most developed non-destructive analytical technique for AgNPs in solution because the change in spectral absorbance on both physical change and behavior of AgNPs in suspension. Thus, the occurrence of AgNPs in solution was investigated spectroscopically using an ultraviolet-visible (UV-vis) spectrophotometer (series Cary 100 UV-Vis spectrophotometer, Agilent, Santa Clara, US) based on their surface plasmon resonance (SPR). Soon after irradiation process, the absorbance UV-vis spectra were measured using a $1 \mathrm{~cm}$ pathlength quartz cuvette at wavelength of 200-800 nm at $1 \mathrm{~nm}$ resolution. The measure spectra was subjected to fitting/deconvultion analysis using Gaussian function.

The colloidal AgNPs was dropped above copper grid and dried, then the morphology of AgNPs were observed under a JEM-1400 transmission electron microscopy (JEOL, Tokyo, Japan) operating at $120 \mathrm{kV}$. Further, the TEM micrograph was processed and analyzed for average particle size and polydispersity index (PDI) using software ImageJ (ver. ImageJ 1.51i, developed by Wayne Rasband, National Institutes of Health, USA). 
Prior to Fourrier transform infrared (FTIR) spectroscopy, the colloids were subjected to lyophilization using freeze-drying instrument (series Beta I, Christ, Osterode am Harz, Germany). Then, the lyophilized samples were dispersed in $\mathrm{KBr}$ powder. The FTIR spectra of samples was measured over spectral range of 4000-400 $\mathrm{cm}^{-1}$ using FTIR spectrophotometer (series IRPrestige21, Shimadzu, Kyoto, Japan).

The $\mathrm{pH}$ change after and before irradiation were measured using a $\mathrm{pH}$ meter (series PB-10 Basic Meter, Sartorius AG, Goettingen, Germany).

\section{Stability of Colloidal AgNPs}

The stability of colloidal AgNPs as a function of time was characterized using particle instability parameter (PIP) based on UV-Vis absorbance spectroscopy $[19,20]$. The UV-vis spectra of samples were recorded and processed as previously described at several time points $(3,6,13$, and 15 day). Based on the peak intensity and wavelength shift, the PIP value is defined as in Eq. (1).

$$
P I P=\sqrt{\left(I^{*}\right)^{2}+\left(\lambda^{*}\right)^{2}}
$$

where $I^{*}$ is a weighted intensity value and $\lambda^{*}$ is weightened wavelength shift. The $\mathrm{I}^{*}$ is defined as in Eq. (2):

$$
I^{*}=\frac{\Delta I}{I_{0}}=\frac{I_{0}-I_{n}}{I_{0}}
$$

where $\Delta \mathrm{I}$ is a change in peak intensity from the reference peak intensity $\left(\mathrm{I}_{0}\right)$ to peak intensity at $\mathrm{n}$ point in time. The weightened wavelength shift is defined as in Eq. (3).

$$
\lambda^{*}=C \Delta \lambda=C\left(\lambda_{0}-\lambda_{n}\right)
$$

where $\lambda_{0}$ is the reference peak wavelength position, $\lambda_{\mathrm{n}}$ is the peak position at $\mathrm{n}$ point in time and $\mathrm{C}$ is a weighting function defined as in Eq. (4).

$$
C=\frac{I_{\text {thresh }}^{*}}{\lambda_{\text {thresh }}^{*}}=\frac{0.1}{10}
$$

such that an unstable system is defined by a $10 \%$ change in intensity or a wavelength shift of $10 \mathrm{~nm}$ $[20,19]$.

\section{RESULTS AND DISCUSSION}

The 1-carragenan solution is colorless and transparent as shown in Fig. 1(a). Interestingly, the irradiated solution remains colorless and transparent after irradiated at doses up to $20 \mathrm{kGy}$.
Previous research reported that the gamma irradiation caused the browning of carrageenan solution [21]. The brown color is related to formation of double bond after main chain degradation and/or hydrogen abstraction [22]. Browning due to gamma irradiation has also been reported for irradiated other polysaccharides, such as alginate [22,23] and chitosan [24,25].

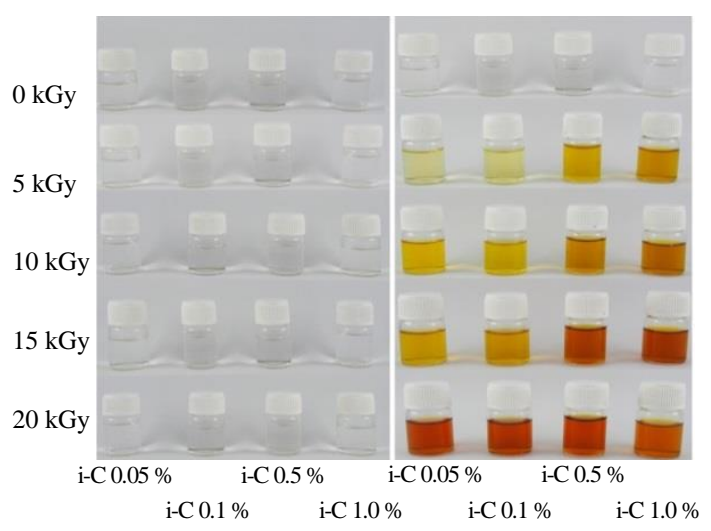

(a)

(b)

Fig. 1. Appearance of irradiated 1-carrageenan solution at various doses and concentration (a) without and (b) with addition of $1 \mathrm{mM} \mathrm{AgNO}_{3}$.

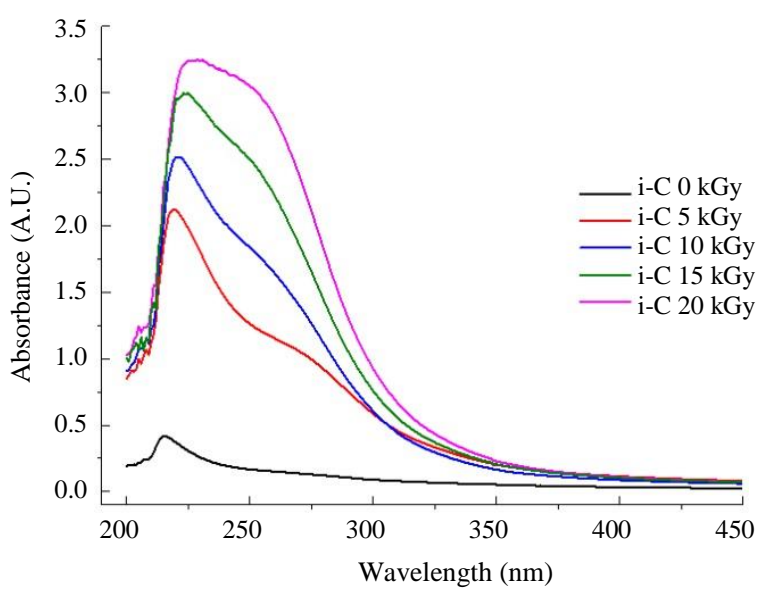

(a)

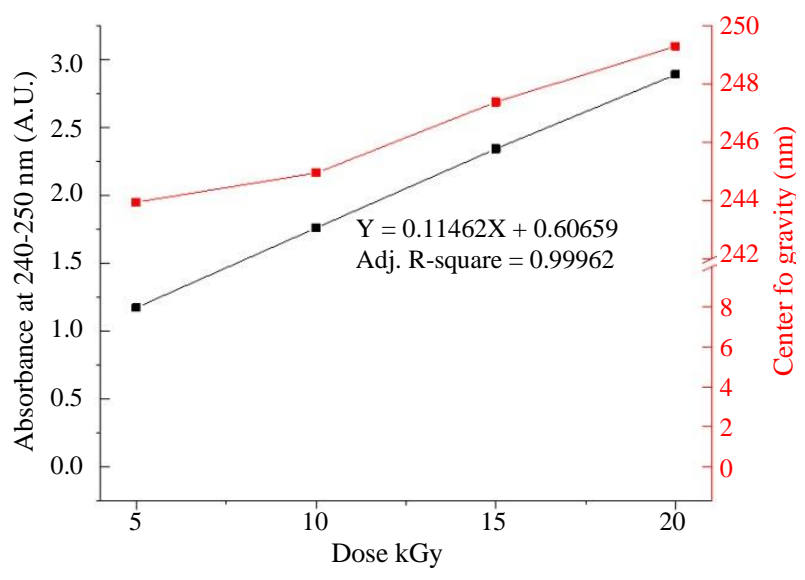

(b)

Fig. 2. (a) UV-vis spectra of t-carrageenan irradiated at various doses and (b) their absorbance intensity at $240-250 \mathrm{~nm}$. 
The formation of double bond can be evidenced by the occurance of absorbance peak at about $265 \mathrm{~nm}$ in UV-vis spectra $[21,22,25]$. Based on Fig. 2(a)-(b) (data shown in suppl. data Table S1), the UV-vis spectra of irradiated samples show a new peak as compared to unirradiated sample, but it is not centered at about $260 \mathrm{~nm}$. The absorption peak is centered at about $247 \mathrm{~nm}$ which is slightly red shifted from 243.94 to $249.29 \mathrm{~nm}$ with increasing irradiation dose. Intensity of the peaks are linearly correlated with increasing dose (adj. $\left.R^{2}=0.996\right)$. This peak is assigned to the formation of carbonyl group which is confirmed by occurrence of new peak at $1721 \mathrm{~cm}^{-1}$ in FTIR spectra of the irradiated samples, as shown in Fig. 3. These results comfirm that double bond linkage is not formed during irradiation under condition in this research, thus the irradiated 1-carragenan solution remains colourless and transparent.

The color change is known to be influenced by type of gases in reaction system. Nagasawa et al. [22] reported that dark brown color under gamma irradiation occurs when polysaccharides solution is irradiated under nitrogen gas and air atmosphere, but little color change is observed on irradiation in the presence of oxygen. Even more, no color change observed when $\mathrm{pH}$ of solution is set below 3.0 at sufficient amount of oxygen [25]. It seems that the absent of brown color in this study is due to the occurance of significant amount of dissolved oxygen within 1 -carragenan solution. The dissolved oxygen is possibly transferred from atmosphere during homogenization of solution with vigorous vortex at room temperature.

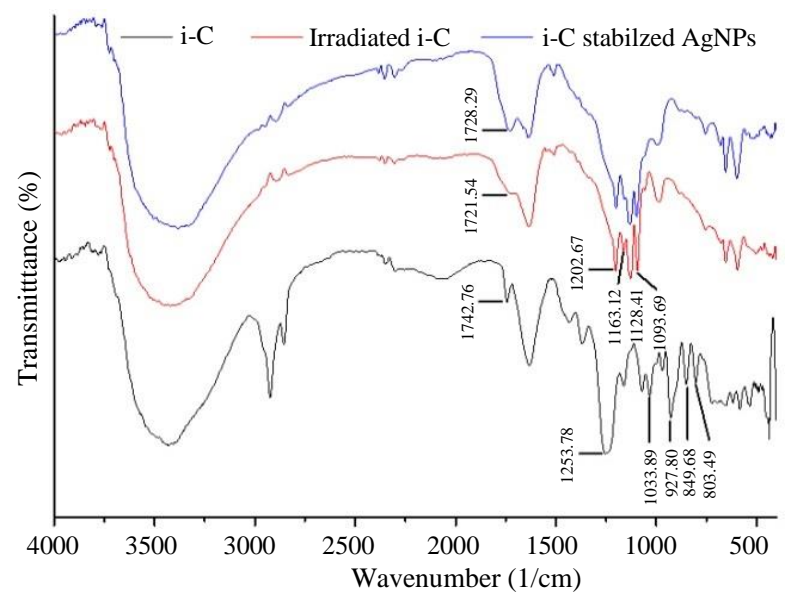

Fig. 3. FTIR spectra of raw 1 -carrageenan, irradiated i-carrageenan, and i-carrageenan supported AgNPs.

Gamma irradiation of $\mathbf{t - c a r r a g e n a n}$ solution containing $\mathrm{AgNO}_{3}$ leads to color change from colorless transparent to yellow-to-brown, as shown in Fig. 1(b). The UV-vis spectra of samples show a new absorption peak which is not observed in unirradiated sample and irradiated samples without addition of $\mathrm{AgNO}_{3}$, as shown in Fig. 4.

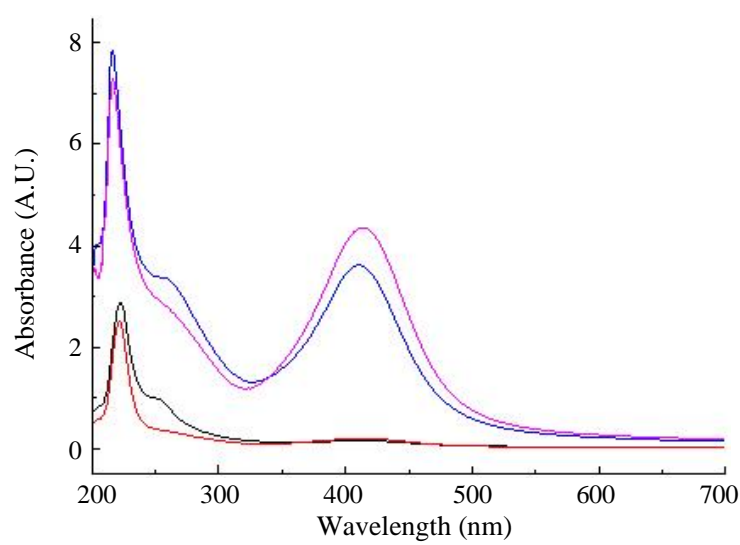

(a)

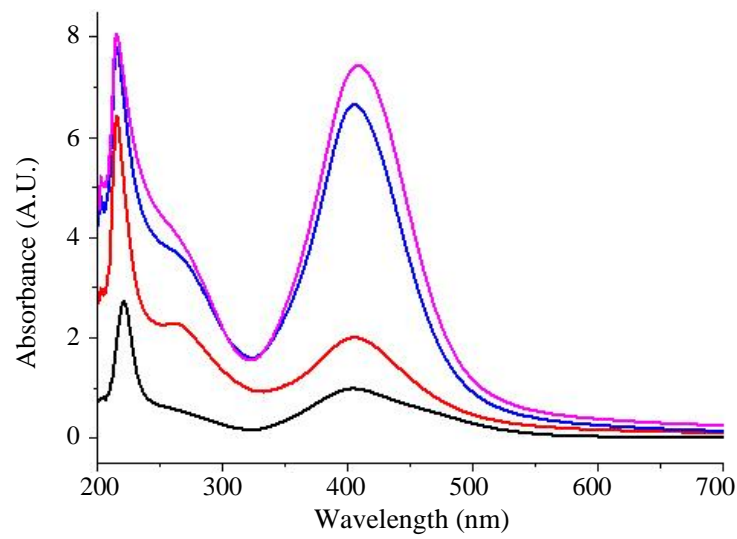

(b)

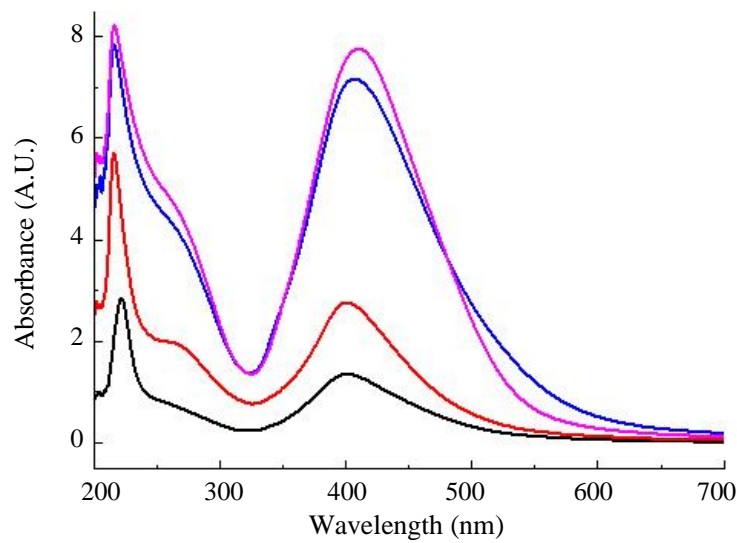

(c)

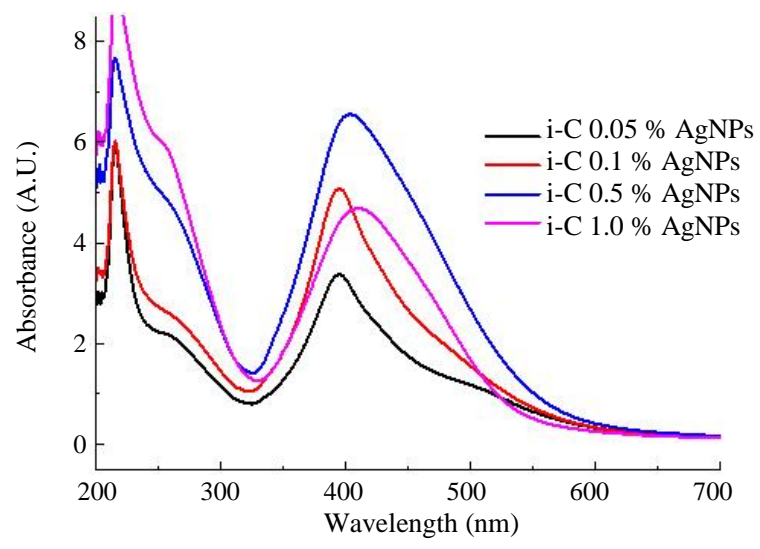

(d)

Fig. 4. UV-vis spectra of 1 -carrageenan supported AgNPs synthesized at dose of (a) 5, (b) 10, (c) 15 and (d) $20 \mathrm{kGy}$. 
The light absorption gives rise to a well-defined band of scattered wavelengths in the visible spectrum specifically at wavelength of about $395-418 \mathrm{~nm}$, in contrast to small nonresonant scatterers that have monotonously increasing scattering cross-section toward shorter wavelength. This peak corresponds to the surface plasmon resonance (SPR) band of silver particles that arise from the coherent oscillation of conduction electron near AgNPs surfaces $[8,18]$.

The occurrence of AgNPs in solution is confirmed by TEM measurement, as shown in Fig. 5 (data shown in suppl. data Fig. S1). The primary morphology of the AgNPs is spherical, while a small portion of AgNPs are rod and triangular. The average particle size is at about 7.6-9.17 $\mathrm{nm}$ which vary slightly in related to i-carrageenan concentration. Most of the particles have a size of below $20 \mathrm{~nm}$; however, several particles at a range of 20-53 $\mathrm{nm}$ are observed. The PDI values of colloidal AgNPs are below 0.5 which indicates that particle size distribution is at monodisperse system. This result confirms that gamma irradiaton can be a convenient means to the synthesis of AgNPs which is in agreement with previous reports [3].

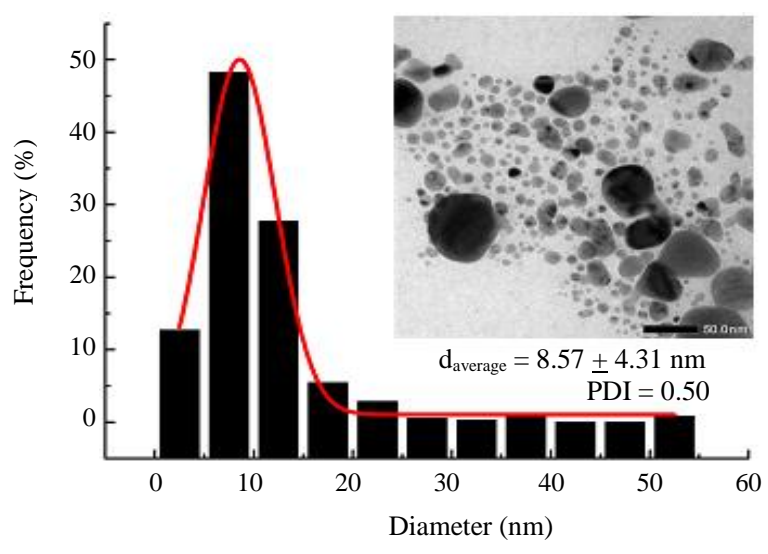

(a)

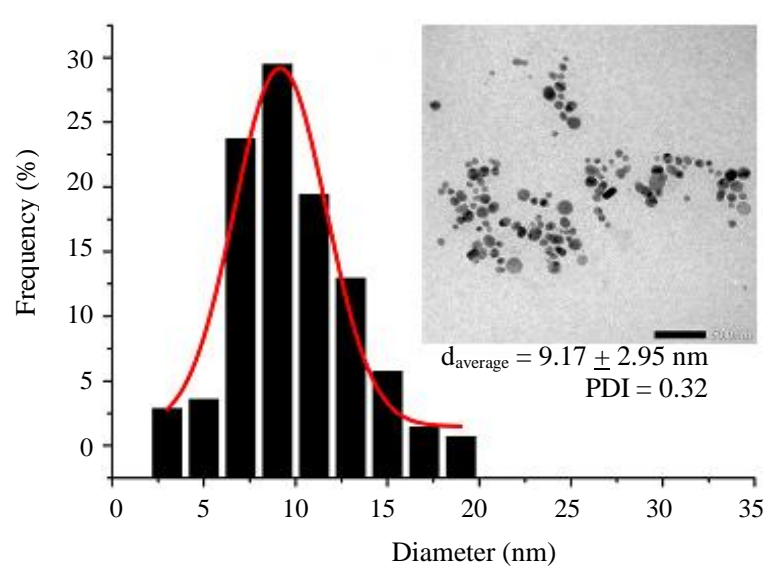

(b)

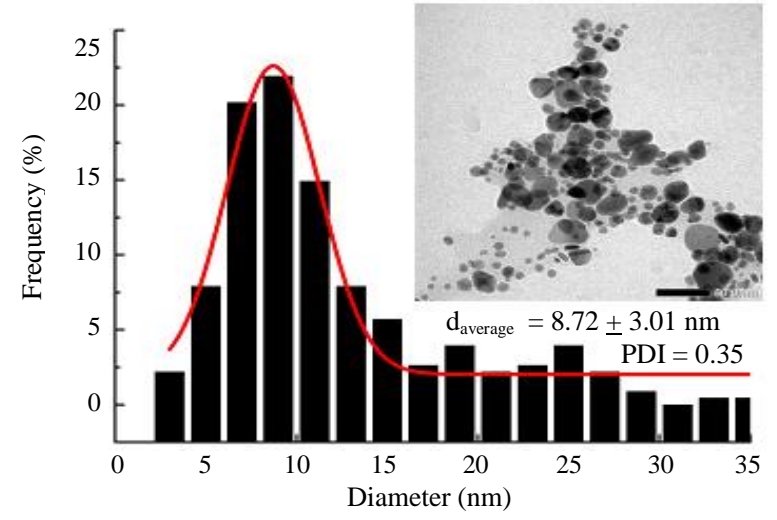

(c)

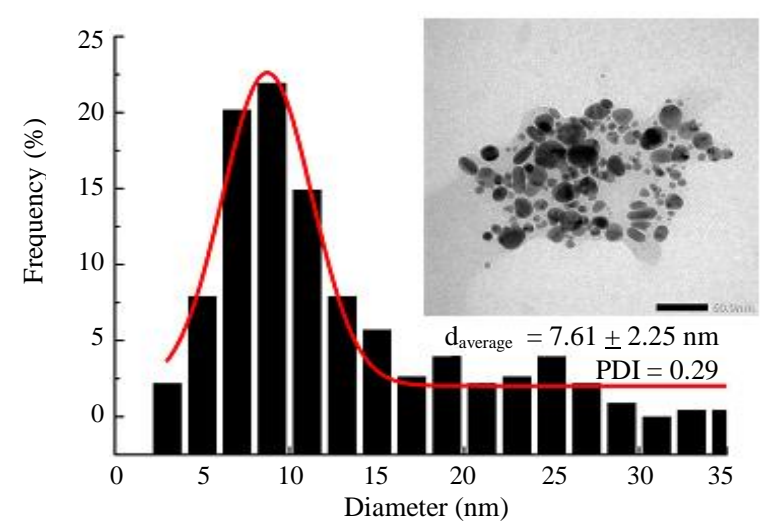

(d)

Fig. 5. TEM micrograph and particle size distribution of AgNPS stabilized in (a) 0.05 , (b) 0.1 , (c) 0.5 , and (d) $1.0 \%$ (w/v) i-carrageenan

The fundamental of gamma radiosynthesis relies on the radiolysis of water into radical species, reduction of $\mathrm{Ag}^{+}$ion by reducing species into uncharged state $\mathrm{Ag}^{0}$ (nucleation stage), and subsequent coalescent of $\mathrm{Ag}^{0}$ to form AgNPs (growth stage). Radiolysis of water generates a number of species with very short half-lifes $\left(\sim 10^{-10}\right)$ through simplified reaction in Eq. (5):

$$
\mathrm{H}_{2} \mathrm{O} \stackrel{\gamma-\mathrm{ray}}{\longrightarrow} \mathrm{OH}^{\bullet}, e_{a q}^{-}, \mathrm{H}^{\bullet}, \mathrm{H}_{2}, \mathrm{H}_{2} \mathrm{O}_{2}, \mathrm{H}^{+}
$$

where solvated electron and hydrogen radicals are strong reducing agent with redox potential of $E^{\circ}\left(\mathrm{H}_{2} \mathrm{O} / e_{\mathrm{aq}}{ }^{-}\right)=-2.87 \mathrm{~V}_{\mathrm{NHE}}$ at $\mathrm{pH} 7$ and $E^{\circ}\left(\mathrm{H}^{+} / \mathrm{H} \bullet\right)=$ $-2.3 \mathrm{~V}_{\mathrm{NHE}}$, respectively. Both species can reduce silver ion by following in Eqs. (6) and (7) [1,2]:

$$
\begin{gathered}
e_{a q}^{-}+A g^{+} \rightarrow A g^{0} \\
H^{\bullet}+A g^{+} \rightarrow A g^{0}
\end{gathered}
$$

Binding energy between two $\mathrm{Ag}^{0}$ dimers or between $\mathrm{Ag}^{0}$ and $\mathrm{Ag}^{+}$is stronger than the binding energy between $\mathrm{Ag}^{0}$ and the solvent. Therefore, once silver atoms are synthesized, they 
tend to interact to form dimers, following in Eqs. (8) and (9) [1,2].

$$
\begin{aligned}
& A g^{0}+A g^{0} \rightarrow A g_{2} \\
& A g^{0}+A g^{+} \rightarrow A g_{2}^{+}
\end{aligned}
$$

Charged dimer clusters are further reduced and act as centers for nucleation, as follows in Eq. (10) [1,2].

$$
A g_{2}^{+}+\mathrm{Ag}_{2}^{+} \rightarrow \mathrm{Ag}_{4}^{2+}
$$

Under continuous gamma irradiation, absorption of $\mathrm{Ag}^{+}$onto the nuclei leads to the formation of clusters, as follows in Eq. (11) [1,2].

$$
A g_{m}+\mathrm{Ag}^{+} \rightarrow A g_{m+1}^{+}
$$

Finally, monodisperse AgNPs are generated by coalescent process, as follows in Eq. (12).

$$
A g_{m+x}^{x+}+A g_{p+y}^{y+} \rightarrow A g_{n+z}^{z+}
$$

where $\mathrm{m}, \mathrm{n}$, and $\mathrm{p}$ represent nuclearitites, while $\mathrm{x}, \mathrm{y}$, and $\mathrm{z}$ are the numbers of associated $\mathrm{Ag}$ ions [1,2]. Various morphologies occur when AgNPs in (9) grow past a critical size [1].

Interestingly, radiosynthesis in this research was conducted under simple condition, i.e., without the use of neurotoxic isopropanol (hydroxyl scavenger) and under atmospheric gases. However, previous researches [1,2] reported that hydroxyl radicals are capable of oxidizing $\mathrm{Ag}^{+}$and/or $\mathrm{Ag}^{0}$ into higher oxidation state. Also, dissolved oxygen can cause oxidation and corrosion to metal nanoparticles $[1,2]$. The reducing condition in this research is related to dual stabilizing/reducing activity of l-carrageenan which would be explained based on SPR spectra of the colloidal AgNPs in Fig. 4.

The SPR is sensitive to changes in the local dielectric environment. Among noble metal nanoparticles, AgNPs has the highest efficiency of plasmon excitation in the visible spectrum due to the different dielectric properties originated from the small overlap between the SPR and the interband transition in silver from $4 d$ electrons to the hybridized $5 \mathrm{sp}$ band that starts at $320 \mathrm{~nm}[9,26]$. There have been many studies that have characterized the good correlation between change in SPR spectral absorbance on both physical change and behavior of AgNPs in suspension [8,9,18], thus UV-vis spectroscopy can be a fast and easy analytical tool to monitor AgNPs properties in the suspension.

As shown in Fig. 4, the UV-vis spectra of all samples have a single SPR peak due to dipolar resonance mode of electron near the surface of AgNPs. Dipolar plasmon resonance only occurs when the dimension of the particles is much smaller than wavelength of incident light, specifically for a diameter of below $67 \mathrm{~nm}$ for AgNPs because all electrons in the entire particle experience a roughly uniform electric fielding [8]. Also, single SPR peak is also only occurs for well-dispersed isolated silver particles suspension [9]. Thus, this phenomena confirm the results of TEM micrograph that silver particles in this study was at nanosize and welldispersed as isolated particles within the suspension. This results indicate that $\mathrm{t}$-carrageenan is a good stabilizer to generate AgNPs via bottom-up approach. The stabilization is related to entrapment of $\mathrm{Ag}^{+}$ions by polar $\mathrm{OH}$ groups and electron-rich oxygen atoms on the r-carrageenan chain when $\mathrm{AgNO}_{3}$ solution is added to 1 -carrageenan solution [14]. During radiosynthesis, the entrapped $\mathrm{Ag}^{+}$ions along i-carrageenan chains were reduced into $\mathrm{Ag}^{0}$ as following $(6,7)$, which sequentially act as passivated nucleating point for growth of well-dispersed isolated nanosilver particles as following (8-12).

Peak intensity increases with increasing 1carrageenan concentration at certain irradiation dose of up to $15 \mathrm{kGy}$, as shown in Fig. 4(a)-(c) (data shown as spectrum 0D in suppl. data Fig. S2-S5). The SPR absorbance correlates to AgNPs concentration and obeys the Lambert-Beer law $[18,27]$. Thus, the result means that concentration of AgNPs in suspension increases with increasing i-carrageenan concentration. Peak intensity also increases with increasing irradiation dose in which maximum value is reached at lower irradiation dose at higher 1-carrageenan (data shown as spectrum 0D in suppl. data Fig. S2-S5). Among all the treatments, the highest value is achieved at treatment of irradiation dose of $10 \mathrm{kGy}$ and 1-carrageenan of $1.0 \%(\mathrm{w} / \mathrm{v})$ which was measured at 7.58 A.U. with FWHM of $82.14 \mathrm{~nm}$. For t-carrageenan of 0.05 and $0.1 \%(\mathrm{w} / \mathrm{v})$, the peak intensity keeps increasing with increasing dose up to $20 \mathrm{kGy}$. This results indicate that i-carrageenan acts not merely as a stabilizing agent, but also as a reducing agent in the preparation of AgNPs. Higher concentration of i-carrageenan and/or increasing gamma irradiation yields more degradation products with reducing activity. The dual stabilizing/reducing activity of carrageenan has been previously reported for microwave-assisted synthesis of AgNPs in which reducing activity is performed by the sulphate groups of i-carrageenan [13]. Under gamma irradiation, the mechanism of reduction activity seems related to antioxidant activity of degradation product of 1-carrageenan. Previous reports [28,29] reported that degradation product of carrageenan shows hydroxyl scavenging, reducing power, and DPPH radical scavenging activity. Specifically for degradation by gamma irradiation, those activities are related to depolymerization which is accompanied by increased reducing ends [28]. 
However, peak intensity decreases after irradiation at 15 and $10 \mathrm{kGy}$ for $\mathrm{t}$-carrageenan of 0.5 and $1.0 \%(\mathrm{w} / \mathrm{v})$, respectively. The decreasing intensity is companied with red-shift of $\lambda_{\text {SPR }}$ as well as broadening of SPR spectra. The broadening and redshift of resonant bands indicates the increases in particle size or agglomeration of nanoparticles. Previous researches [5,8,9] have reported that a red-shift and broadening of the dipolar resonance is observed in large particles along with the appearance of higher-order of SPR modes due to radiative losses. When dimension of AgNPs increases, light cannot polarize homogenously and the field is no longer uniform throughout the NP, which results in phase retardation effect $[8,9]$. Shorter 1-carrageenan chains at higher irradiation doses provides lower steric stabilizing activity for AgNPs. This result suggests that an optimum irradiation dose need to be determined for balancing the optimum trade-off between stabilizing and reducing activity.

Previous study [21] reported that important functional groups are still present in the FTIR spectra of irradiated $\mathrm{t}$-carrageenan after irradiation at $100 \mathrm{kGy}$. In this study, functional groups related to ester sulphate disappear after gamma irradiation as shown in Fig. 3. The unirradiated 1-carrageenan shows characteristic peaks related to ester sulphate at $1253 \mathrm{~cm}^{-1}$, C-O linkage of 3,6-anhydro-Dgalactose at 1033 and $27 \mathrm{~cm}^{-1}$, and galactose 4sulphate at $849 \mathrm{~cm}^{-1}$. e uniradiated sample also shows a peak related to 3,6-anhydro-D-galactose-2sulphate at $803 \mathrm{~cm}^{-1}$ which is the characteristic and the distinctive properties of 1-carrageenan. All the characteristic peaks are absent in FTIR spectra of irradiated sample with and without addition of $\mathrm{AgNO}_{3}$. It seems that irradiation at oxygenic condition causes massive removal of sulphate groups. The removal of sulphate groups changes the $\mathrm{pH}$ of the solution from neutral to acidic, which decreases with increasing irradiation doses and t-carrageenan concentration, as shown in Fig. 6. The differences on degree of 1-carrageenan degradation and $\mathrm{pH}$ suspension would alter the stability of AgNPs in suspension.

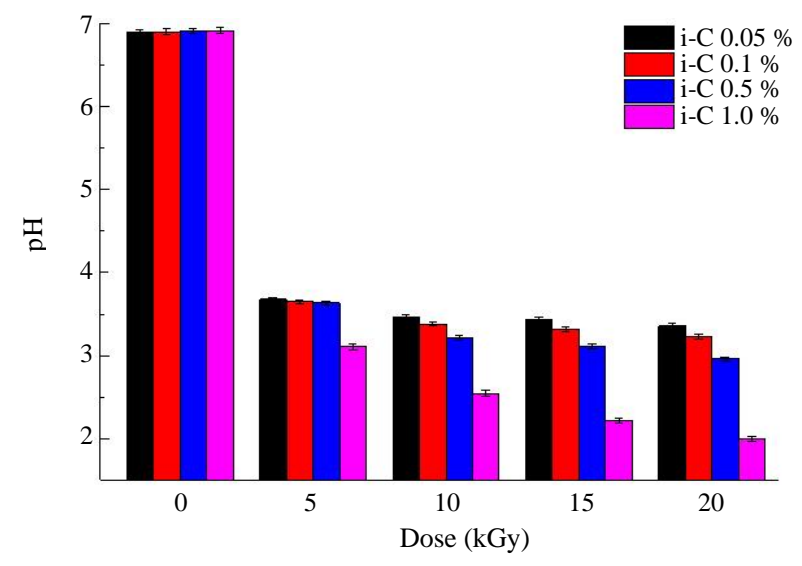

Fig. 6. $\mathrm{pH}$ of 1-carrageenan irradiated at various doses.

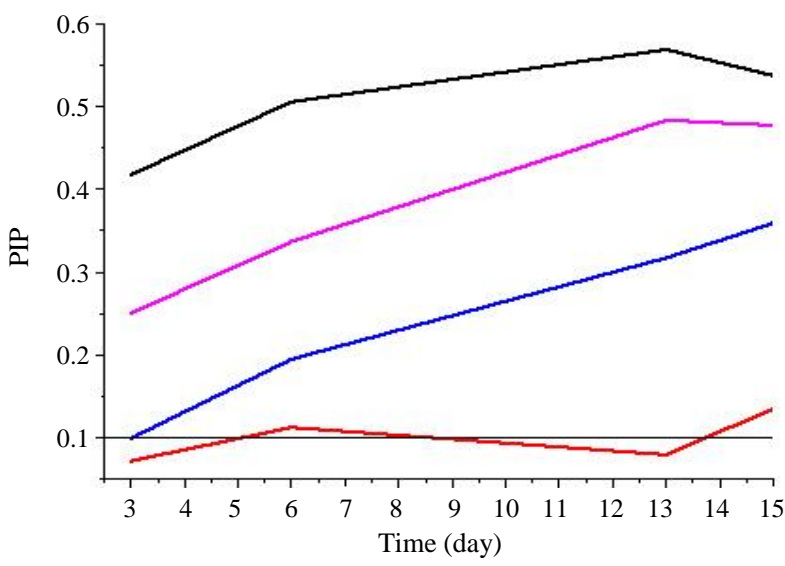

(a)

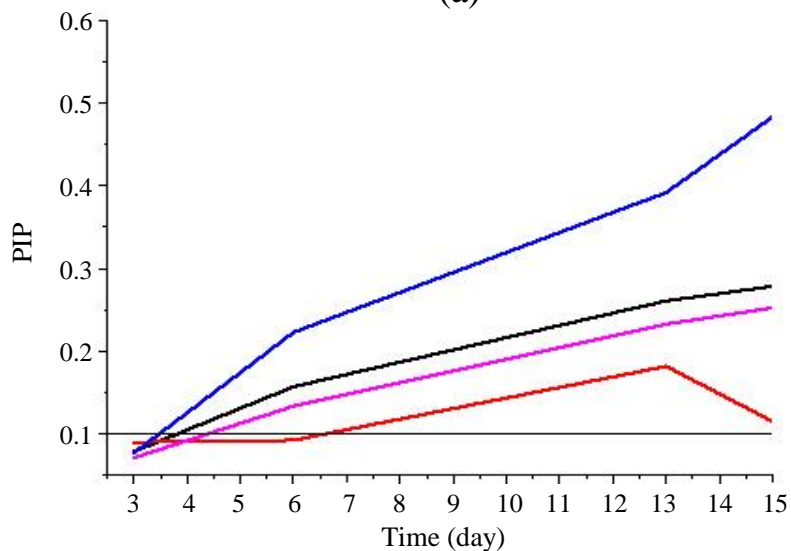

(b)

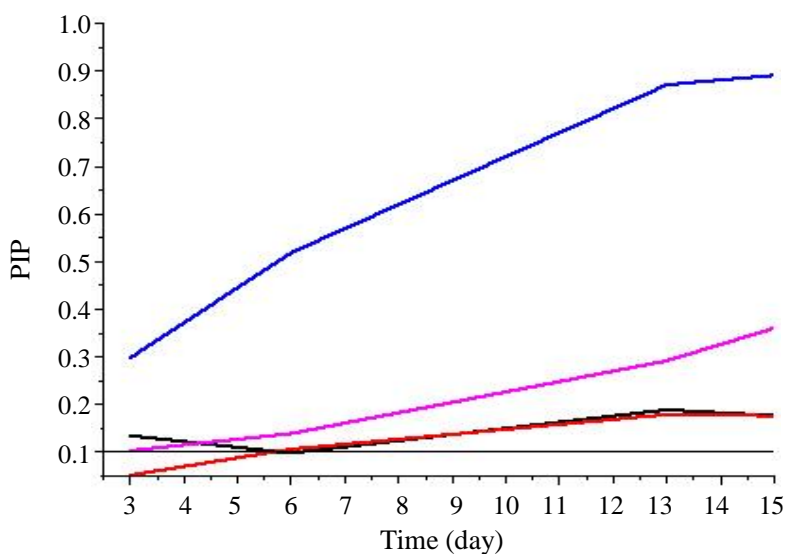

(c)

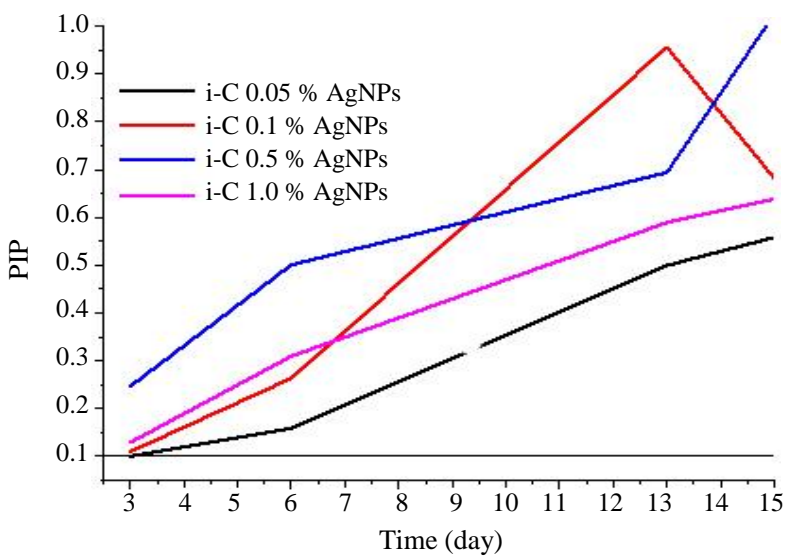

(d)

Fig. 7. Particle instability parameter of 1 -carrageenan supported AgNPs synthesized at $\gamma$-ray dose of (a) 5, (b) 10, (c) 15 and (d) $20 \mathrm{kGy}$ at function of time. 
The potential for application of AgNPs is strongly dependent on their stability against aggregation due to a strong correlation between size and biological activity of AgNPs [30-32]. As shown in Fig. 7, most of colloidal AgNPs are unstable as their PIP are higher than the cut-off value of 0.1 . Stable AgNPs are only observed for 3 days for all AgNPs synthesized at doses of $10 \mathrm{kGy}$ and for AgNPs synthesized at $5 \mathrm{kGy}$ and $10 \mathrm{kGy}$ stabilized in $0.1 \%(\mathrm{w} / \mathrm{v}) \mathrm{t}$-carrageenan. For colloidal AgNPs synthesized at a dose of $5 \mathrm{kGy}$, increasing PIP value by time sourced is mainly from decreasing intensity of SPR peak (data shown in suppl. data Table S2-S5). This results indicate the decreasing AgNPs concentration due to oxidative dissolution of AgNPs at low $\mathrm{pH}$ of colloidal AgNPs as follows in Eqs. (13) and (14).

$$
\begin{aligned}
& 4 \mathrm{Ag}+\mathrm{O}_{2} \rightarrow 2 \mathrm{Ag}_{2} \mathrm{O} \\
& \mathrm{Ag}_{2} \mathrm{O}+2 \mathrm{H}^{+} \rightarrow 2 \mathrm{Ag}^{+}+\mathrm{H}_{2} \mathrm{O}
\end{aligned}
$$

where metallic silver oxidized by dissolved oxygen and subsequently reacts with protons [33]. Meanwhile, the shape of dipolar vibration peaks are invariable with small change in the $\lambda_{n}$. It seems that agglomeration does not occur due to spacious interparticle distant at low AgNPs concentration.

For colloidal AgNPs synthesized at higher doses, increasing PIP value by time sourced occurs from both decreasing intensity and wavelength-shift of the $\lambda_{n}$ (data shown in suppl. data Table S2-S5). Meanwhile, the shape of SPR vibration peaks are broadening accompanied with the formation of shoulder peak indicating the agglomeration of AgNPs [8,9]. Agglomeration seems related to degradation of 1 -carrageenan into shorter chains at higher irradiation doses which lowering their steric stabilizing activity. Low $\mathrm{pH}$ also contributes to the increasing agglomeration rate of AgNPs [33,34]. Therefore, $\mathrm{pH}$ adjustment is one of among the critical process parameters to maintain the stability of colloidal AgNPs.

\section{CONCLUSION}

In summary, gamma irradiaton has been used as a means to synthesize colloidal AgNPs. The nanoparticles are spheroid in shape which is monodisperse with average particle size below $10 \mathrm{~nm}$. By using i-carrageenan as a stabilizing agent, the radiosynthesis in this research was conducted under atmospheric gases and without isopropanol addition due to dual stabilizing/reducing activity of i-carrageenan. Highest AgNPs concentration is found at irradiation dose of
$10 \mathrm{kGy}$ and $\mathrm{t}$-carrageenan of $1.0 \%$ (w/v). However, the 1 -carrageenan concentration and irradiation dose need to be optimized to balance the trade-off between reducing activity and stabilizing activity. Also, further work is needed to improve stability of radiosynthesized colloidal AgNPs stabilized in i-carrageenan solution.

\section{ACKNOWLEDGMENT}

Authors acknowledge The Research and Development Center for Marine and Fisheries Product Processing and Biotechnology, Ministry of Marine and Fisheries Affair, Indonesia for kindly providing the i-carrageenan for this research. We also acknowledge Drs. Erizal, APU, and Dr. Darmawan Darwis (Center for Application of Isotope and Radiation, National Nuclear Energy Agency, Indonesia) for helpful discussion and encouragement.

\section{AUTHOR CONTRIBUTION}

Dian Pribadi Perkasa contributed as the main contributor of the paper. All Authors read and approved the final version of the papers.

\section{REFERENCES}

1. S. M. Ghoreishian, S.-M. Kang, G. S. R. Raju et al., Chem. Eng. J. 360 (2019) 1390.

2. K. Čubová and V. Čuba, Radiat. Phys. Chem. Oxf. Engl. 158 (2019) 153.

3. A. M. S. Hosny, M. T. Kashef, S. A. Rasmy et al., Adv. Nat. Sci: Nanosci. Nanotechnol. 8 (2017) 1.

4. A. M. Elbarbary and N. M. El-Sawy, Polym. Bull. 74 (2017) 195.

5. M. T. S. Alcântara, N. Lincopan, P. M. Santos et al., Radiat. Phys.Chem. 169 (2020) 108777.

6. N. Saha, P. Trivedi and S. D. Gupta, J. Cluster Sci. 27 (2016) 1893.

7. S. Yeasmin, H. K. Datta, S. Chaudhuri et al., J. Mol. Liq. 242 (2017) 757.

8. N. G. Bastús, J. Piella and V. Puntes, Langmuir 32 (2016) 290.

9. V. Amendola, O. M. Bakr and F. Stellacci, Plasmonics 5 (2010) 85.

10. S. H. Lee and B.-H. Jun, Int. J. Mol. Sci. 20 (2019) 865.

11. L. N. Shirokova, A. A. Revina, V. A. Aleksandrova et al., Inorg. Mater. Appl. Res. 7 (2016) 730. 
12. R. Madhukumar, K. Byrappa, Y. Wang et al., Radiat. Eff. Defects Solids 172 (2017) 11.

13. Y. Wang, X. Dong, L. Zhao et al., Nanomater. 10 (2020) 83.

14. N. H. Azeman, N. Arsad and A. A. A. Bakar, Sens. 20 (2020) 3924.

15. P. C. Y. Ng, B. J. Long, W. T. Davis et al., Intern. Emerg. Med. 13 (2018) 375.

16. C. Wang, D. Samaha, S. Hiremath et al., Syst. Rev. 7 (2018) 250.

17. H. R. Greene and M. D. Krasowski, Toxicol. Rep. 7 (2020) 81.

18. G. Moreno-Martin, M. E. León-González and Y. Madrid, Talanta 188 (2018) 393.

19. S. Mourdikoudis, R. M. Pallares and N. T. K. Thanh, Nanoscale 10 (2018) 12871.

20. A. Rohaizad, S. Shahabuddin, M. M. Shahid et al., J. Environ. Chem. Eng. 8 (2020) 103955.

21. L. Relleve, N. Nagasawa, L. Q. Luan et al., Polym. Degrad. Stab. 87 (2005) 403.

22. N. Nagasawa, H. Mitomo, F. Yoshii et al., Polym. Degrad. Stab. 69 (2000) 279.
23. H. L. A. El-Mohdy, Arabian J. Chem. 10 (2017) S431.

24. S. I. Jeong, S.-C. Park, S.-J. Park et al., Int. J. Nanomed. 13 (2018) 525.

25. W. Yue, Carbohydr. Polym. 101 (2014) 857.

26. S. I. Mogal, D. O. Shah, T. Mukherjee et al., ACS Omega 3 (2018) 12802.

27. M. Dell'Aglio, V. Mangini, G. Valenza et al., Appl. Surf. Sci. 374 (2016) 297.

28. L. V. Abad, L. S. Relleve, C. D. T. Racadio et al., Appl. Radiat. Isot. 79 (2013) 73.

29. A. M. Suganya, M. Sanjivkumar, M. N. Chandran et al., Biomed. Pharmacother. 84 (2016) 1300.

30. C. H. N. Barros, S. Fulaz, D. Stanisic et al., Antibiot. 7 (2018) 69.

31. Q. Lin, J. Y. C. Lim, K. Xue et al., VIEW 1 (2020) e16.

32. Z. A. Ratan, M. F. Haidere, M. Nurunnabi et al., Cancer 12 (2020) 855.

33. I. Fernando and Y. Zhou, Chemosph. 216 (2019) 297.

34. M. Ndikau, N. M. Noah, D. M. Andala et al., Int. J. Anal. Chem. 2017 (2017) 1. 